\title{
Three-year results from a preclinical implantation study of a long-term resorbable surgical mesh with time-dependent mechanical characteristics
}

\author{
H. Hjort • T. Mathisen • A. Alves • G. Clermont • \\ J. P. Boutrand
}

Received: 27 June 2011 / Accepted: 18 September 2011/Published online: 5 October 2011

(C) Springer-Verlag 2011

\begin{abstract}
Purpose The purpose of this study was to evaluate the biocompatibility, local tissue effects and performance of a synthetic long-term resorbable test mesh $\left(\right.$ TIGR $^{\circledR}$ Matrix Surgical Mesh) compared to a non-resorbable polypropylene control mesh following implantation in a sheep model. Methods Full-thickness abdominal wall defects were created in 14 sheep and subsequently repaired using test or control meshes. Sacrifices were made at 4, 9, 15, 24 and 36 months and results in terms of macroscopic observations, histology and collagen analysis are described for 4, 9, 15, 24 and 36 months.

Results The overall biocompatibility was good, and equivalent in the test and control meshes while the resorbable mesh was characterized by a collagen deposition more similar to native connective tissue and an increased thickness of the integrating tissue. The control polypropylene mesh provoked a typical chronic inflammation persistent over the 36-month study period. As the resorbable test mesh gradually degraded it was replaced by a newly formed collagen matrix with an increasing ratio of collagen type I/III, indicating a continuous remodeling of the collagen towards a strong connective tissue. After 36 months, the test mesh was fully resorbed and only microscopic implant residues could be found in the tissue. Conclusions This study suggests that the concept of a long-term resorbable mesh with time-dependent mechanical
\end{abstract}

H. Hjort $(\bowtie) \cdot$ T. Mathisen

Novus Scientific, Rapsgatan 25, 75450 Uppsala, Sweden

e-mail: henrik.magnusson@novusscientific.com

A. Alves - G. Clermont - J. P. Boutrand

Biomatech-NAMSA, Lyon, France characteristics offers new possibilities for soft tissue repair and reinforcement.

Keywords Preclinical - Hernia - Mesh · Implant . Resorbable · Absorbable · Degradable $\cdot$ Soft tissue . Collagen

\section{Introduction}

Surgical meshes have been used routinely for various soft tissue reinforcement applications since the introduction of Dacron $^{\circledR}$ (polyethylene terephthalate, PET) and Marlex ${ }^{\circledR}$ (polypropylene, PP) in the late 1950s [1, 2]. For hernia repair, polypropylene meshes in particular have been considered the standard material for fabrication of prosthetic meshes used for the tension-free hernia repair described by Lichtenstein et al. [3] in 1989 as well as for other surgical techniques. The inflammatory responses to the more frequently used mesh products have been investigated on human explants by Klinge et al. [4], who confirm the view that mesh materials used for soft tissue reinforcement elicit a chronic inflammatory response that is persistent over time. Histological observations describe an implanted permanent mesh, typically characterized by a foreign body granuloma adjacent to the mesh fiber and a surrounding collagen capsule that shields the host from the foreign material. It is reasonable to assume that this chronic inflammatory process impairs normal wound healing and tissue regeneration [5] but it may still be acceptable in the light of the proposed function of permanent implants, the purpose of which being mainly restoration of mechanical functionality.

In order to improve the outcome of the use of permanent meshes it has further been suggested and shown that 
decreasing the amount of permanent material implanted can be beneficial for patients [6-8]. A plausible next step would be the use of resorbable or degradable mesh products that function as scaffolds and are gradually replaced by functional connective tissue. Products made from enzymatically degradable collagen of xenogenic or allogenic origin have been used for this purpose but with questionable outcomes in terms of benefit versus the high cost [9]. Synthetic meshes made of glycolide and a small percentage of trimethylene carbonate or lactide are characterized by a fast degradation leading to a fast decline in mechanical properties. Nonetheless, such meshes have been used successfully in applications where no real load support is required over any significant period of time, such as spleen preservation $[10,11]$ or for temporary support of abdominal wall reconstructions [12]. Initial short-term results of hernia repair using quick-degrading meshes have also recently been published [13].

A new approach to abdominal wall repair and soft tissue reinforcement makes use of a mesh that maintains adequate mechanical strength and subsequently resorbs completely over a longer period of time, allowing the body to rebuild a more functional connective tissue. Early preclinical experiences have been published by both de Tayrac et al. $[14,15]$ and Klinge et al. [16] but no successful long-term follow-up has been made available. The purpose of this study was to evaluate the mechanical performance and histopathological response of a synthetic long-term resorbable mesh with a novel time-dependent mechanical behavior, TIGR ${ }^{\circledR}$ Matrix Surgical Mesh (test), compared to a nonresorbable polypropylene mesh (control) during longterm functional implantation in sheep abdominal wall.

\section{Materials and methods}

TIGR $^{\circledR}$ Matrix Surgical Mesh is a macroporous multifilament surgical mesh, knitted from two different synthetic resorbable fibers, possessing different degradation characteristics. The first, fast-degrading fiber, is a co-polymer of glycolide, lactide and trimethylene carbonate. The second, slow-degrading fiber, is a co-polymer of lactide and trimethylene carbonate. Both fibers degrade by bulk hydrolysis once implanted, resulting in decreasing strength retention followed by mass loss of the fibers. The mesh maintains more than $50 \%$ of its initial strength for 6 months and is completely resorbed after approximately 3 years. When the fast-degrading fiber starts to lose its mechanical strength, at around 2 weeks following implantation, the mesh changes its knitted configuration and gradually becomes more mechanically compliant. This time-dependent mechanical characteristic is thought to enable an increasing load transfer from the mesh to the new connective tissue that integrates with it, thereby mechanically stimulating increased load-directed collagen remodeling [17].

The study was approved by a local ethics committee and performed in accordance with FDA and OECD Good Laboratory Practices (GLP) requirements. All animals received standard hay and municipal water ad libitum throughout the study and were identified by individual ear tags. Each of 14 female sheep was anesthetized, clipped, scrubbed with povidone iodine and placed in a dorsal decubitus position. A medial incision of the abdominal skin was performed and the external surface of the abdominal wall was exposed. Four full-thickness, $3 \times 3 \mathrm{~cm}$ defects were created within the abdominal musculature. The abdominal muscular layer was carefully removed leaving the peritoneum intact. On each defect, a test or control mesh of $8 \times 8 \mathrm{~cm}$ was placed in an onlay position and secured with interrupted non-absorbable sutures (Fig. 1) (Prolene, Ethicon, Norderstedt, Germany). The skin was closed by continuous intracutaneous absorbable sutures (Vicryl, Ethicon). For each observation time point, two animals received three test and one control product each, and one animal received only test products. For the 24 and 36 months time points, only two animals carrying three test and one control mesh were kept for each time point. One animal was kept in reserve.

During a 10-day observation period following surgery, the animals were observed daily for any clinical abnormalities. During this period, the animals were housed in a controlled environment. Following the observation period, all animals were transferred to a farm setting where the environmental conditions were uncontrolled. All conditions conformed to the European requirements for farm animals (EEC Directive 86/609).

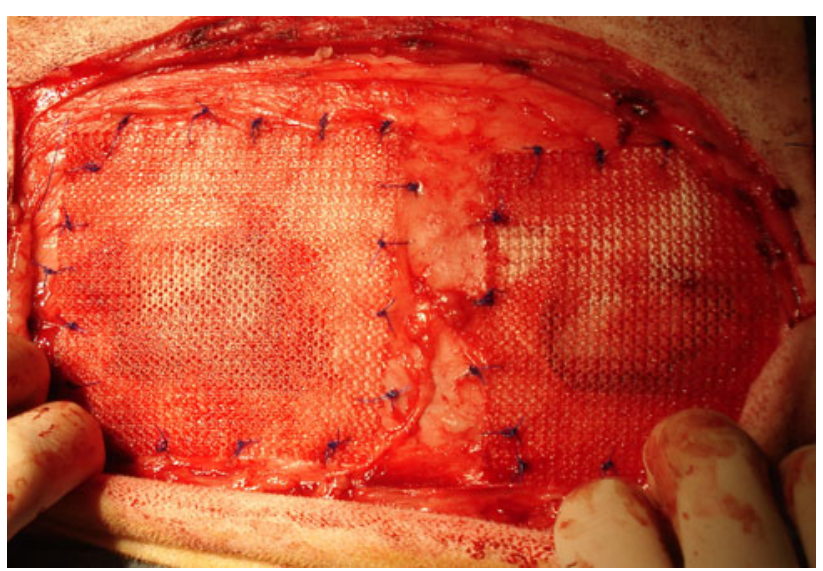

Fig. 1 Implantation of $8 \mathrm{~cm}$ square meshes over $3 \mathrm{~cm}$ square fullthickness defects (peritoneum left intact) in the abdominal wall of sheep 
Animals were sacrificed using a lethal injection of pentobarbital solution at designated intervals $(4,9,15,24$ and 36 months), and macroscopic observations of the sites were performed. For all sites, macroscopic degradation of the meshes, local signs of irritation (hemorrhage, necrosis, exudate, neovascularization, degradation of the mesh) and any adhesions between the mesh and the abdominal structures were scored. All the test and control meshes were harvested. After labeling and fixation in $10 \%$ buffered formalin solution, specimens were dehydrated in alcohol solutions of increasing concentration, cleared in xylene and embedded in paraffin. Sections were prepared and stained with Masson's trichrome and hematoxylin-eosin-safranin (HES). Histological slides were observed under a Leica DMR microscope, equipped with a Leica DFC 420C digital camera. Qualitative and semi-quantitative histological evaluation of the local tolerance and material degradation of each implanted site was performed by a qualified pathologist. The scale used was 0: absent, 1: slight, 2: moderate, 3: marked, 4: severe.

Test and control specimens along with an untreated sheep abdominal wall were also sampled for scanning electron microscopy (SEM) at 4 and 9 months. These samples were post-fixed in buffered glutaraldehyde solution after fixation in buffered formalin solution, then dehydrated in $20 \%$ acetone solution and treated with gold palladium. SEM was performed using a Hitachi S800 microscope under $15 \mathrm{keV}$. Pictures were taken in the areas of interest. SEM was conducted to evaluate tissue integration of the devices as well as collagen penetration, distribution, maturity and material degradation.

In order to differentiate between collagen types I and III, paraffin sections of $5 \mu \mathrm{m}$ in thickness were stained according to the technology of Junqueira [18] and analyzed under cross-polarization microscopy (CPM). Collagen type I appeared as red-orange shades whereas collagen type III displayed pale-green color. Sections of rat spleen and lymph nodes, rich in collagen III [19] were prepared under the same conditions and served as controls to accurately set the angle of polarization before grading collagen type III. Abundant in normal skin tissue, collagen type I was recognized easily in the implanted sites.

\section{Results}

Post-surgical complications

No defect recurrences were observed throughout the course of the study. One sheep developed an infection at the implanted site and was euthanized and replaced by a reserve animal. Within the first month after surgery, abdominal swelling was observed in eight sheep that otherwise showed good general health status. The swelling was likely due to seroma formation provoked by the rather extensive dissection needed to fit four implants on each animal. Five of these sheep showed hyperthermia and received antibiotics for 4 days. Spontaneous regression was observed, except for one animal on which punctures were performed under sterile conditions. After these punctures, spontaneous regression was observed.

\section{Macroscopic evaluation}

At all time points, the macroscopic evaluations showed no local adverse effects for either the test or control meshes, and no biologically significant differences were ever observed macroscopically. For both test and control, the mesh-tissue complex showed early neovascularization and the meshes were well integrated into fibrous connective tissue. Some differences between individual animals were noted at all time points. In general, the control mesh was easier to locate due to its stiffness as compared to the test mesh, where the mesh-tissue complex was difficult to differentiate from the intact abdominal wall upon palpation of the sites. This was more evident at the 24- and 36-month sacrifices as the polypropylene control mesh was stiff, yet not well integrated and the tissue layers were easily separated from the mesh following explantation. As expected, the control mesh never showed any signs of degradation whereas the first fiber of the test meshes was completely degraded after 4 months and all test meshes were macroscopically undetectable at 36 months.

\section{Histologic evaluation}

There were no histological signs, at any time, of residual infection or necrosis for either test or control meshes. At 4 months, the control mesh showed no signs of alteration, and elicited a chronic inflammatory reaction (Fig. 2) characterized by a moderate grade of phagocytotic cells infiltrating the mesh, and a slight grade of neutrophils, plasma cells and lymphocytes. A moderate grade of fibroplasia was observed peripherally and a large amount of mature collagen of marked grade was observed between the fibers, but also surrounding the mesh.

After 4 months, the fast-degrading fiber of the test mesh was histologically absent. The slow-degrading multifilament fibers of the test mesh were surrounded by a moderate grade of fibrous tissue and induced an inflammatory reaction qualitatively and quantitatively similar to that observed with the control mesh, except for a higher amount of collagen deposition with a more variable maturity and an occasionally greater lymphocytic reaction (Fig. 3a). A moderate degree of neovessel formation could be observed in the surrounding tissue as well as adjacent to the mesh 


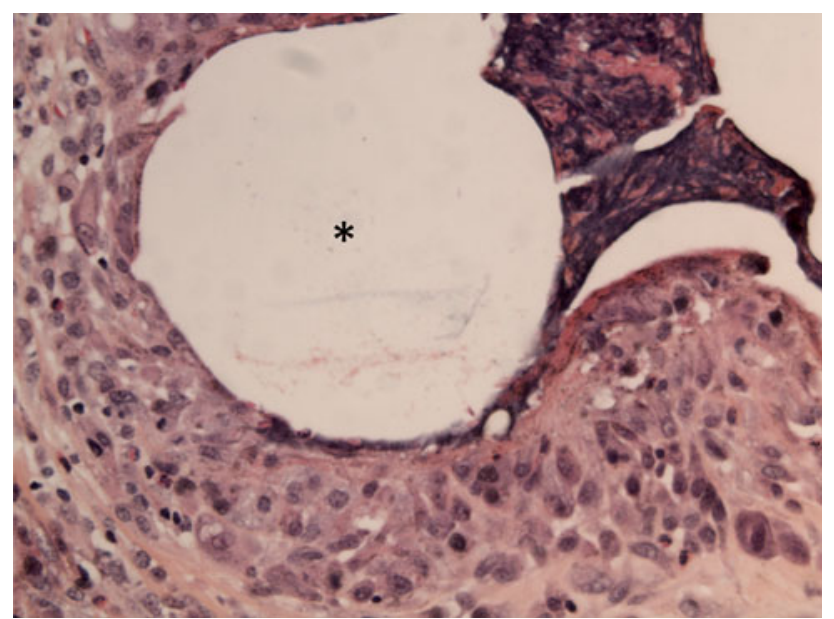

Fig. 2 Hematoxylin-eosin-safranin (HES)-stained micrograph showing the inflammatory reaction surrounding a polypropylene monofilament fiber (asterisk) at 4 months following implantation

fibers. A small proportion of the test sites showed slight to moderate grade of interfascicular fatty tissue deposition, which was considered to be incidental (Fig. 3b).

The histological appearance of both test and control meshes was similar at 4 and 9 months. At 9 months, test sites were still characterized by a higher amount of collagen deposition, ranging from marked to severe, leading to thicker connective tissue, infiltrating and surrounding the mesh (Fig. 4a). Histological analysis of the collagen types showed that the test implants were associated with a higher rate of connective tissue maturation when compared to control implants after 9 months. The quality of the collagen was deemed closer to the native abdominal wall within the test implant sites.

At 15 and 24 months, the control mesh showed a slight chronic inflammatory reaction. A moderate grade of colonization and slight grade of encapsulation was evident, along with a marked grade of integration of the fibers (Fig. 4b). The test mesh induced an inflammatory reaction qualitatively and quantitatively higher than that of the control mesh, due mainly to a higher recruitment of phagocytotic cells around and between the multifilaments (Fig. 4c). This is part of the degradation process [20] as the fibers constituting the multifilament displayed evidence of degradation under polarized light. Collagen deposition seemed to be an ongoing process 15 months post implantation.

The overall thickness of mature collagen deposition was higher (score 2.8) than in the control group (score 2) at 24 months. The fibers constituting the multifilaments of the test mesh displayed an advanced stage of degradation under polarized light, and fibers of decreased size were found commonly in the cytoplasm of macrophages and giant cells indicating an active phagocytotic process (Fig. 5a).

The test mesh was fully degraded at 36 months and replaced by a connective tissue of variable thickness, and only a few implant residues were observed (Fig. 5b). The control mesh showed no signs of degradation at 36 months after implantation. The inflammatory response to the test mesh had decreased significantly between the 24 and 36 months time points and was clearly lower compared to the control group at 36 months. Only a few macrophages and giant cells showing the intracytoplasmic presence of material debris could be observed. The general connective tissue layer formed was thicker around the test mesh as compared to the control.

\section{Scanning electron microscopy}

The thickness of the native abdominal wall was approximately $5 \mathrm{~mm}$, with normal layers of muscle, fat and connective tissue. The control mesh showed a wall thickness of approximately $1.3 \mathrm{~mm}$ after 9 months, whereas the test specimen revealed a newly formed abdominal wall of approximately $6 \mathrm{~mm}$. At 4 and 9 months, both test and control specimens showed a well-integrated mesh without signs of encapsulation. The collagen surrounding the filaments was mature with marked signs of remodeling and maturity. These findings were consistent at 24 and 36 months.

\section{Collagen analysis}

In the early phase of wound healing, granulation tissue rich in collagen type III, which is characterized by weak tensile
Fig. 3 HES-stained micrographs at 4 months following implantation. a Inflammatory reaction, neovessels (hat) and collagen generation around test mesh multifilament fibers (asterisk). b Interfascicular fatty tissue between collagen layers surrounding the test mesh
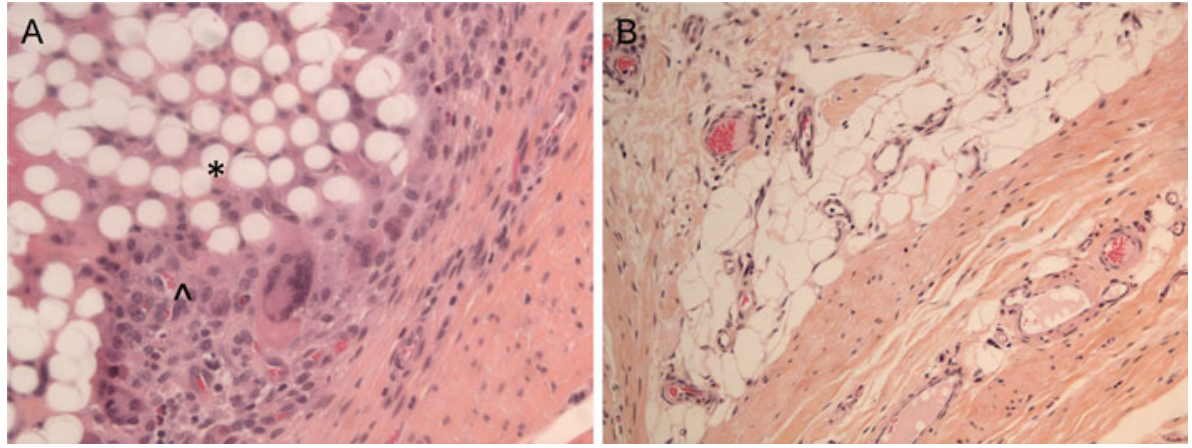
Fig. 4 HES-stained micrographs. a Test mesh at 9 months with fibers (asterisk) integrated into thick connective tissue. b Control mesh at 15 months with slightly encapsulated fibers (hat). c Test mesh at 15 months with phagocytotic cells surrounding the fibers (asterisk)
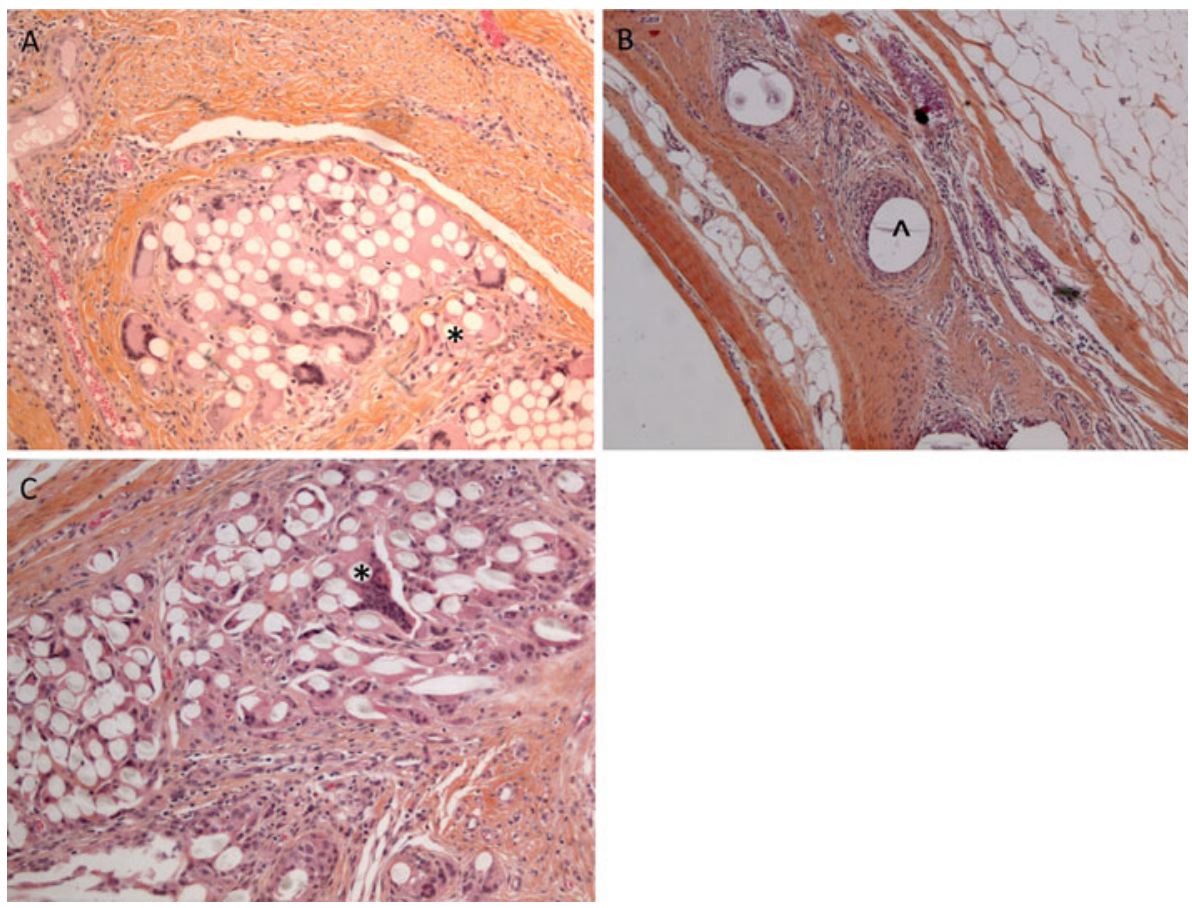

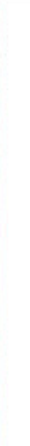

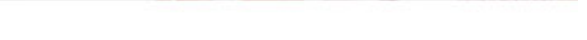

Fig. 5 a Phagocytotic activity around degraded fibers (asterisk) of the test mesh at 24 months following implantation (HES staining). b Masson-trichrome staining showing fiber remnants (hat) of the test mesh at 36 months following implantation
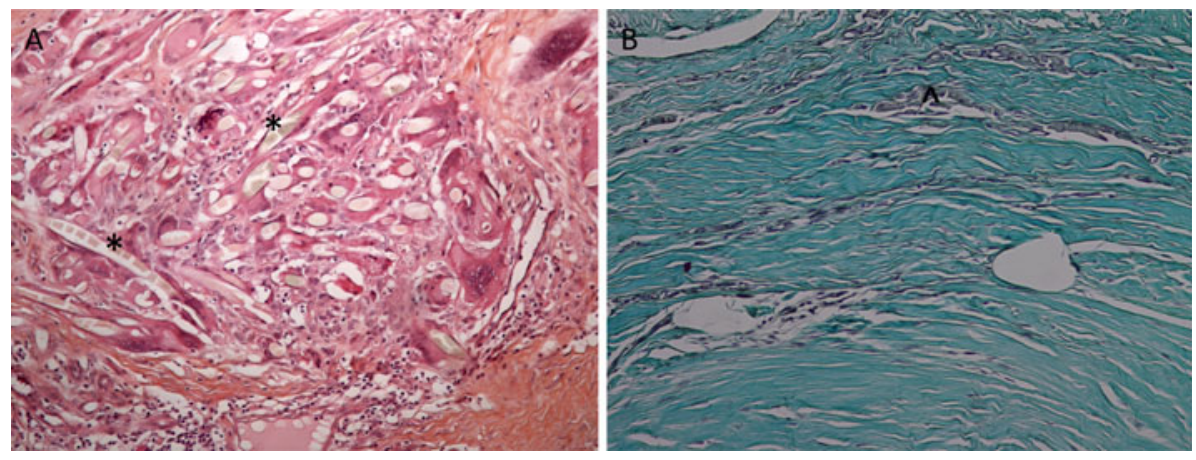

properties, is deposited. Later in the wound healing process collagen type III is replaced by collagen type I characterized by increased tensile properties [21, 22]. In an attempt to determine the ratio between collagen types I and III, selected histology sections from the 9-month observation period were stained according to the method described by Junquiera [18]. The result revealed a slightly higher ratio of collagen type I at the test sites associated with a higher rate of connective tissue maturation when compared to the control implants.

Based on the above results, a more thorough histomorphometric analysis of the collagen ratio in some of the slides originating from 15, 24 and 36 months observations was performed.

Specimens were taken only from animals carrying both test and control meshes in order to compensate for individual variability.

In both histology and SEM analysis, the test sites displayed a gradual increase in collagen content within the extracellular matrix between 15 and 36 months post implantation (Fig. 6a). This is in sharp contrast to the control sites, which showed a gradual decrease in the collagen content within the extracellular matrix formed around and within the control mesh. One explanation for this could be the fat tissue that progressively infiltrated the extracellular matrix over time.

For the test sites the collagen was found to integrate well within the mesh after 24 months and could also be found between individual fiber bundles as well as surrounding the mesh. At 36 months the mesh was no longer visible and the resorbed fiber bundles were fully replaced by new collagen-rich tissue, which could explain the increase in collagen content found within the extracellular matrix.

The ratio of collagen I/III within the test sites increased over time (Fig. 6b), indicating a continuous remodeling of the ingrown collagen towards a strong connective tissue. The control site showed little or no remodeling after 15 months of implantation, with less collagen type I 

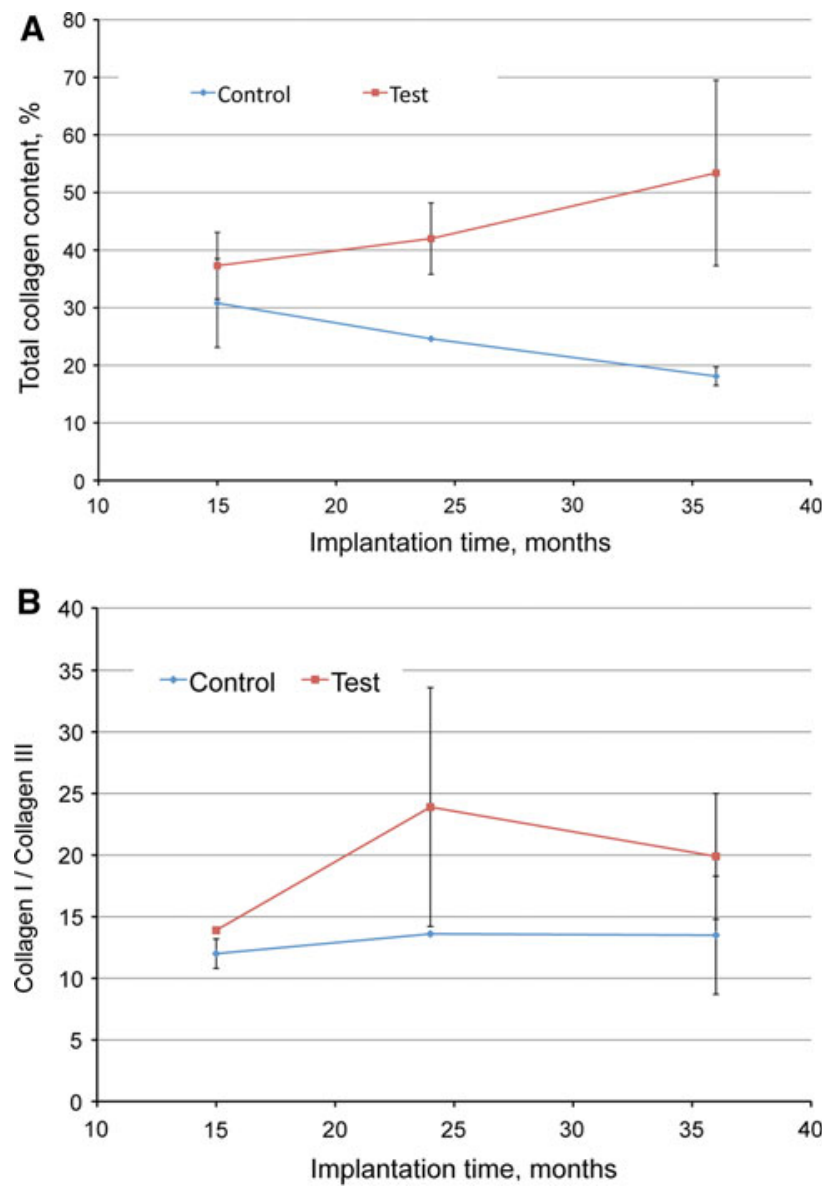

Fig. 6 a Total collagen content as a function of time following implantation. b Collagen type I/III ratio as a function of time following implantation (symbols mean values, bars standard deviation)

deposited within the extracellular matrix surrounding the control mesh as opposed to the test site.

\section{Discussion}

This study, which to the best of our knowledge is the longest preclinical evaluation of surgical meshes conducted to date, verifies previous knowledge on the long-term tissue response to polypropylene. The control mesh elicited an acute inflammatory response that is persistent over time, and that turns into a chronic inflammation characterized by foreign-body granulomas surrounding the fibers and a layer of mature collagen, effectively screening the foreign material from the surrounding tissue. Following explantation at 24 and 36 months, the polypropylene mesh was macroscopically not well integrated and was still stiff, and was shown histologically to be encapsulated by wellorganized and mature collagen. This corresponds well to previous knowledge of how a host organism protects itself from a foreign object [23].

TIGR $^{\circledR}$ Matrix Surgical Mesh-the world's first synthetic long-term resorbable surgical mesh-exhibits a timedependent mechanical behavior. In this study it elicited an acute and medium-term inflammatory response similar to that of polypropylene, with the important difference that the inflammation declined after 24 months and was practically absent after 36 months. As the matrix degraded completely over 36 months and the inflammatory reaction decreased, a well-remodeled and durable connective tissue replaced the mesh. The collagen layer surrounding the test mesh was thicker and more similar to native connective tissue in its structure as compared to the control mesh.

Considering the comparably low number of control specimens, conclusions should be drawn with caution. It is however evident that there is a substantial difference in the long-term outcome between the test and control mesh when looking at the quantity and quality of the connective tissue that integrates with, or in the case of the test mesh, ultimately replaces the product. We believe these results are due to the differences in mechanical characteristics. A rigid mesh product will absorb the loads by itself and any new connective tissue infiltrate will lack mechanical stimulation, which has been shown to have effects on connective tissue stability [17, 24]. The mechanical behavior of the test mesh was designed to be as closely matched to the tissues where it is to be implanted as practically possible, so that bodily loads could be progressively transferred to the new tissue that is deposited around the mesh and between its fibers. Further studies will be required to investigate this concept of mechanical stimulation of soft tissues more thoroughly. This could potentially lead to a better understanding of soft tissue defects in general, and ultimately to better surgical treatment methods.

Some soft tissue defects that may require surgical intervention, recurrent hernias in particular, are understood to be the result of inadequate ability to form, or maintain adequately, strong connective tissue. Since this study was performed in healthy animals, collagen deficiencies have not been taken into account, which raises the interesting and important question of whether the results shown here would reproduce in a patient population with known collagen diseases or deficiencies. Measuring collagen expression over time following soft tissue repair using TIGR $^{\circledR}$ Matrix in a clinical setting may lead to increased knowledge of this complex situation.

Finally, as no defect recurrences were noted in either the test or control group, and since TIGR ${ }^{\circledR}$ Matrix substantially loses its mechanical strength after 6 months following implantation, the restored tissue is evidently strong enough to carry the abdominal loads in this sheep model. Despite the ongoing discussion regarding the pathology of hernias 
and other soft tissue defects, we conclude that our findings suggest that TIGR $^{\circledR}$ Matrix Surgical Mesh may represent a new way of utilizing long-lasting, resorbable synthetic polymers that could be of great benefit in soft tissue repair or reinforcement.

Acknowledgments The authors would like to thank Per Busch and Kristofer Rubin at the Department of Medical Biochemistry and Microbiology, Uppsala University for valuable contributions to this study. This study was funded by Novus Scientific.

\section{References}

1. Wolstenholme JT (1956) Use of commercial dacron fabric in the repair of inguinal hernias and abdominal wall defects. AMA Arch Surg 73:1004-1008

2. Usher FC, Ochsner J, Tuttle LL Jr (1958) Use of marlex mesh in the repair of incisional hernias. Am Surg 24:969-974

3. Lichtenstein IL, Shulman AG, Amid PK, Montllor MM (1989) The tension-free hernioplasty. Am J Surg 157:188-193

4. Klinge U, Klosterhalfen B, Müller M, Schumpelick V (1999) Foreign body reactions to meshes used for the repair of abdominal wall hernias. Eur J Surg 165:665-673

5. Szpaderska AM, DiPietro LA (2005) Inflammation in surgical wound healing: friend or foe? Surgery 137:571-573

6. O'Dwyer PJ, Kingsnorth AN, Molloy RG, et al. (2005) Randomized clinical trial assessing impact of a lightweight or heavyweight mesh on chronic pain after inguinal hernia repair. $\mathrm{Br}$ J Surg 92:166-170

7. Post S, Weiss B, Willer M et al (2004) Randomized clinical trial of lightweight composite mesh for Lichtenstein inguinal hernia repair. Br J Surg 91:44-48

8. Smietański M, Bury K, Smietańska IA et al. (2011) Five-year results of a randomized controlled multi-centre study comparing heavy-weight knitted versus low-weight, non-woven polypropylene implants in Lichtenstein hernioplasty. Hernia. doi: 10.1007/s10029-011-0808-y

9. Blatnik J, Jin J, Rosen M (2008) Abdominal hernia repair with bridging acellular dermal matrix —an expensive hernia sac. Am J Surg 196:47-50

10. Lange DA, Zaret P, Merlotti GJ, Robin AP, Sheaff C, Barrett JA (1988) The use of absorbable mesh in splenic trauma. J Trauma 28:269-275
11. Delany HM, Rudavsky AZ, Lan S (1985) Preliminary clinical experience with the use of absorbable mesh splenorrhaphy. J Trauma 25:909-913

12. Jernigan TW, Fabian TC, Croce MA et al (2003) Staged management of giant abdominal wall defects. Ann Surg 238:349-357

13. Efthimiou M, Symeonidis D, Koukoulis G et al (2011) Open inguinal hernia repair with the use of a polyglycolic acid-trimethylene carbonate absorbable mesh: a pilot study. Hernia 15:181-184

14. de Tayrac R, Chentouf S, Garreau H et al (2008) In vitro degradation and in vivo biocompatibility of poly(lactic acid) mesh for soft tissue reinforcement in vaginal surgery. J Biomed Mater Res B Appl Biomater 85:529-536

15. de Tayrac R, Letouzey V, Garreau H et al (2010) Tissue healing during degradation of a long-lasting bioresorbable gamma-raysterilised poly(lactic acid) mesh in the rat: a 12-month study. Eur Surg Res 44:102-110

16. Klinge U, Schupelick V, Klosterhalfen B (2001) Functional assessment and tissue response of short- and long-term absorbable surgical meshes. Biomaterials 22:1415-1424

17. Rubert JW, Hallab NJ (2005) Strain-controlled enzymatic cleavage of collagen in loaded matrix. Biochem Biophys Res Commun 336:483-489

18. Junquiera LC, Cossermelli W, Brentani R (1978) Differential staining of collagens type I, II and III by Sirius red and polarization microscopy. Arch Histol Jpn 41:267-274

19. Poirier J, Ribadeau-Dumas J-L, Catala M, André J-M (1999) Histologie Moléculaire: Texte et Atlas. 1st edn. Masson, Paris

20. Middleton JC, Tipton AJ (2000) Synthetic biodegradable polymers as orthopedic devices. Biomaterials 21:2335-2346

21. Bailey AJ, Sims TJ, Le Lous M, Bazin S (1975) Collagen polymorphism in experimental granulation tissue. Biochem Biophys Res Commun 66:1160-1165

22. Thermann H, Frerichs O, Holch M, Biewener A (2002) Healing of achilles tendon, an experimental study: part 2-histological, immunohistological and ultrasonographic analysis. Foot Ankle Int 23:606-613

23. Katou F, Ohtani H, Nagura H, Motegi K (1998) Procollagenpositive fibroblasts predominantly express fibrogenic growth factors and their receptors in human encapsulation process against foreign body. J Pathol 186:201-208

24. Chiquet M, Gelman L, Lutz R, Maier S (2009) From mechanotransduction to extracellular matrix gene expression in fibroblasts. Biochim Biophys Acta 1793:911-920 